\title{
Attention-Deficit/Hyperactivity Disorder in Childhood Is Associated with Cognitive Test Profiles in the Geriatric Population but Not with Mild Cognitive Impairment or Alzheimer's Disease
}

\author{
N. Ivanchak, ${ }^{1}$ E. L. Abner, ${ }^{1}$ S. A. Carr, ${ }^{1}$ S. J. Freeman, ${ }^{1}$ \\ A. Seybert, ${ }^{1}$ J. Ranseen, ${ }^{2}$ and G. A. Jicha ${ }^{1,3}$ \\ ${ }^{1}$ Sanders-Brown Center on Aging and the University of Kentucky Alzheimer's Disease Center, \\ University of Kentucky College of Medicine, Lexington, KY 40536, USA \\ ${ }^{2}$ Department of Psychiatry, University of Kentucky College of Medicine, Lexington, KY 40509, USA \\ ${ }^{3}$ Department of Neurology, University of Kentucky College of Medicine, Lexington, KY 40536, USA
}

Correspondence should be addressed to G. A. Jicha, gajich2@email.uky.edu

Received 5 January 2011; Revised 12 May 2011; Accepted 2 June 2011

Academic Editor: Darlene V. Howard

Copyright (@ 2011 N. Ivanchak et al. This is an open access article distributed under the Creative Commons Attribution License, which permits unrestricted use, distribution, and reproduction in any medium, provided the original work is properly cited.

\begin{abstract}
The frequency of ADHD in the aging population and its relationship to late-life cognitive decline has not been studied previously. To address this gap in our understanding, the Wender-Utah ADHD Rating scale (WURS) was administered to 310 geriatric subjects with cognitive status ranging from normal cognition to mild cognitive impairment to overt dementia. The frequency of WURS-positive ADHD in this sample was 4.4\%. WURS scores were not related to cognitive diagnoses, but did show nonlinear associations with tasks requiring sustained attention. The frequency of ADHD appears stable across generations and does not appear to be associated with MCI or dementia diagnoses. The association of attentional processing deficits and WURS scores in geriatric subjects could suggest that such traits remain stable throughout life. Caution should be considered when interpreting cognitive test profiles in the aging population that exhibit signs and symptoms of ADHD, as attentional deficits may not necessarily imply the existence of an underlying neurodegenerative disease state.
\end{abstract}

\section{Introduction}

Attention-deficit hyperactivity disorder (ADHD) is a common learning disability in children [1-7]. Symptoms of ADHD include inattention, hyperactivity, and impulsivity $[2,5]$. About $1-9.5 \%$ of children are estimated to suffer from ADHD [1-3, 5, 8, 9]. Previous studies have suggested that ADHD is increasing in the United States [2, 9]. Factors including increased television and video game use and a diet defined by excessive sugar, high-fructose corn syrup, and preservatives have all been implicated as possible mechanisms leading to ADHD [4, 10-12]. Genetic predisposition to ADHD has also been suggested by several groups and could play a role beyond that of environmental factors in the development of ADHD [13-18].

The long-term consequences of ADHD in the geriatric population and the possible association of ADHD with cognitive decline in older adults are unknown. ADHD persisting into adulthood has been well documented [6, 8, 19-27]. It is possible that early learning disabilities could influence later life cognitive function and be associated with latelife neurocognitive disorders such as Alzheimer's disease [28]. In fact, individuals with a history of ADHD have a higher prevalence of comorbid psychiatric disorders such as antisocial disorders, mood and anxiety disorders, and substance abuse disorders when they reach adulthood [20, 25]. Such comorbidity could contribute to cognitive dysfunction in older adults meeting diagnostic criteria for mild cognitive impairment (MCI), Alzheimer's disease (AD), and other forms of dementia. Indeed, cognitive dysfunction in adults with a diagnosis of ADHD in childhood has been reported by many groups [29-31]. As AD affects over 5 million Americans and is the most common neurodegenerative disease in the world [32], it is important to understand the full spectrum of possible risk factors for this devastating disease. 
ADHD has not previously been studied as a risk factor for or associated feature of sporadic $\mathrm{AD}$ in the geriatric population.

The current study plans to address the gap in knowledge about both the prevalence of childhood ADHD in the geriatric population and the association between ADHD and late life cognitive functioning. We hypothesize that the prevalence of childhood ADHD will be increased in the subset of older adults experiencing late-life cognitive decline manifest as the development of MCI or AD. To test this hypothesis, we used the Wender-Utah Rating Scale (WURS) [33] to retrospectively identify possible cases of childhood ADHD in 310 elderly participants with either a cognitive diagnosis of normal, MCI, mild Alzheimer's disease, or other dementia.

\section{Method}

2.1. Subjects. Study participants were drawn from the University of Kentucky Alzheimer's Disease Center longitudinal research program. They were predominantly Caucasian and between the ages of 62 and 91 years old (Table 1). Cohort participants had detailed cognitive function testing annually and had neurologic and physical examinations biannually or annually. Individuals with a history of substance abuse (including alcohol); major head injury; major psychiatric illness; medical illnesses that are nonstable, impairing, or that have an effect on the CNS; chronic infectious diseases; stroke or TIA; encephalitis; meningitis; or epilepsy are not recruited into the cohort. The mental status testing of our subjects has been described previously [34]. Diagnosis was determined by consensus agreement of a panel including the examining neurologist, neuropsychologist, social worker, and other support staff. This study was approved by the University of Kentucky, College of Medicine Institutional Review Board.

2.2. Neuropsychological and Rating Scale Assessments. The Wender-Utah ADHD scale [33] uses 25 characteristics of ADHD to retrospectively identify suspected cases of childhood ADHD. Participants were asked to consider each question independently and rate how often they experienced the problems on a Likert-type scale scored from 0 (not at all/slightly) to 4 (very much). The total was calculated and a positive diagnosis for presumptive ADHD was assessed at a score of 36 or above that has previously shown to be a reliable estimate for ADHD tendencies and possible DSM-IV diagnosis [33]. Those subjects with a diagnosis of MCI or AD were also provided a second survey for their informant/study partner to complete for response comparison. Subject and informant completed survey results were significantly but only moderately correlated $(r=0.37, P=0.0027$, Spearman correlation). To maintain uniformity in data collection and analysis between all subjects, irrespective of cognitive status, only subject responses were used for the overall data analysis. Substitution of informant versus subject responses did not change the results of the statistical associations or models presented below.

Neuropsychological test variables used in the present analyses include Mini-Mental State Examination (MMSE)
[35], Clinical Dementia Rating scale global \& sum of boxes scores [36], Category Fluency (animal naming), Trailmaking tests A \& B, WAIS Digit-Symbol substitution, Wechsler Logical Memory delayed recall, and the Wechsler Adult Intelligence Scale-revised (WAIS-R) forward and reverse digit span tests. Only scores from the last evaluation prior to implementation and collection of WURS scores were used in the present analysis.

2.3. Statistical Analysis. Standard comparative statistics were used to analyze the data including Chi-square for nominal data, $t$-tests (Student's or Satterthwaite's as appropriate) for continuous variables, and Mann-Whitney $U$-tests for ordinal variables. Performance on neuropsychological tests was compared using multiple linear regression modeling where the test score was the dependent variable, and age, sex, education, cognitive status (normal versus not normal), and ADHD status (WURS-positive versus WURS-negative) were independent variables. The level for statistical significance was set at $P<0.02$ to reduce the likelihood of Type I error given the multiple comparisons made in Tables 1-3; however, the level for statistical significance was retained at $P<0.05$ in the exploratory analysis of nonlinear modeling of the association of animal fluency, WAIS digit span forward, and WURS scores presented in Figure 1. LOESS plots were created as linear regressions with PROC SGPLOT in SAS 9.2. PROC SGPLOT determined the optimal smoothing parameter. For the Animals plot, the smoothing parameter was 0.936. For the Digit Forward plot, the smoothing parameter was 0.335 .

\section{Results}

The WURS was mailed to 687 subjects spanning the cognitive continuum and 320 of these $(46.6 \%)$ responded. A small number $(n=10)$ of the respondents returned only partially completed surveys that precluded formal analysis, resulting in a total sample of $n=310$ used in the present analysis. Subjects who did not respond were similar in age and gender to responders but were less educated $(16.4 \pm 3.4$ years versus $14.4 \pm 2.6$ years, Satterthwaite's $t$-test, 648.1 d.f., $P<0.0001$ ). There was a higher proportion of cognitively impaired subjects (i.e., consensus diagnosis was not "Normal") among those who did not respond as might be expected $\left(\chi^{2}=88.1\right.$, 1 d.f., $P<0.0001)$. The cognitively impaired subjects studied included 42 with MCI (18 non-amnestic and 24 amnestic presentations), 6 who were cognitively impaired but failed to meet current consensus criteria for MCI, and 19 demented subjects.

Demographic variables between WURS categories $(\geq 36$, $<36)$ are shown in Table 1. A presumptive diagnosis of ADHD (as assessed by the WURS) was found in 10 cognitively normal and 3 cognitively impaired subjects $(4.4 \%$ of the total respondents). The groups did not significantly differ on age, sex, years of education, or cognitive diagnosis.

Using standard parametric and nonparametric comparative techniques, WURS-positive, presumptive-ADHD participants did not differ significantly from non-ADHD 
TABLE 1: Demographic and clinical variables in presumptive positive and negative childhood ADHD subjects as determined by Wender-Utah Rating Scale score.

\begin{tabular}{|c|c|c|c|}
\hline Parameter & ADHD positive $(n=13)$ & ADHD negative $(n=297)$ & $P$ value \\
\hline Age (mean years $\pm S D)$ & $74.3 \pm 7.4$ & $77.9 \pm 7.5$ & $0.09^{\dagger}$ \\
\hline Gender $(\mathrm{M}: \mathrm{F})$ & $5: 8$ & $103: 194$ & $0.78^{\ddagger}$ \\
\hline Cognitive status (Normal: Impaired) & $10: 3$ & $233: 64$ & $0.90^{\ddagger}$ \\
\hline Education $($ mean $\pm S D)$ & $15.7 \pm 3.3$ & $16.5 \pm 2.6$ & $0.31^{\dagger}$ \\
\hline \multicolumn{4}{|l|}{ CDR Sum of boxes (median) } \\
\hline Normal cognition & 0.0 & 0.0 & $0.20^{\S}$ \\
\hline Impaired cognition & 3.5 & 0.5 & $0.03^{\S}$ \\
\hline \multicolumn{4}{|l|}{ CDR Global score (median) } \\
\hline Normal cognition & 0.0 & 0.0 & $0.28^{\S}$ \\
\hline Impaired cognition & 1.0 & 0.5 & $0.37^{\S}$ \\
\hline
\end{tabular}

${ }^{\dagger}$ Student's $t$-test, 308 d.f.; ${ }^{\ddagger} \chi{ }^{2}$ test, 1 d.f.; ${ }^{\S}$ Mann-Whitney $U$-test.

TABLE 2: Mean neuropsychological test scores ( \pm SEM) adjusted for age, sex, education, and cognitive status in presumptive positive and negative childhood ADHD subjects as determined by Wender-Utah Rating Scale score.

\begin{tabular}{lccc}
\hline Neuropsychological test scores & ADHD positive $(n=13)$ & ADHD negative $(n=297)$ & $P$ value \\
\hline Folstein MMSE & $27.5 \pm 0.6$ & $27.6 \pm 0.2$ & 0.84 \\
Animal Fluency & $15.8 \pm 1.4$ & $18.7 \pm 0.4$ & 0.055 \\
Trailmaking test A & $45.8 \pm 4.7$ & $46.2 \pm 1.2$ & 0.93 \\
Trailmaking test B & $117.7 \pm 12.2$ & $110.3 \pm 3.1$ & 0.55 \\
WAIS-R Digit-Symbol Substitution & $38.9 \pm 2.8$ & $42.2 \pm 0.7$ & 0.25 \\
WMS Logical Memory Delayed & $10.0 \pm 1.1$ & $10.0 \pm 0.3$ & 0.94 \\
WAIS-R Digit Span Forward & $8.5 \pm 0.5$ & $9.2 \pm 0.1$ & 0.14 \\
WAIS-R Digit Span Backward & $6.5 \pm 0.6$ & $6.8 \pm 0.2$ & 0.55 \\
\hline
\end{tabular}

Abbreviations: MMSE: Mini-Mental State Examination; CDR: Clinical Dementia Rating scale; WAIS-R: Wechsler Adult Intelligence Scale-Revised; WMS: Wechsler Memory Scale.

participants on any of the neuropsychological measures when the means were adjusted for age, sex, education, and cognitive status (Table 2). These results remained consistent when only the data from cognitively normal subjects were considered (Table 3).

These data demonstrated trends for associations with the animal fluency task and WAIS digit span forward that prompted further analysis. Scatter plots with LOESS smooths were constructed to test the assumption of a linear versus nonlinear relationship between WURS scores and performance on neuropsychological test measure performance, and the results were suggestive of a nonlinear relationship on several of the measures (Figure 1). Regression models for the normals only, using WURS score as a quadratic term, demonstrate that the WURS-squared was a significant predictor of performance on animal naming $(P=0.030)$ and WAIS digit span forward (0.037) even after controlling for age and education (gender proved not to be significant in these models, data not shown). The relationship of WURS scores with WAIS digit span backward was not significant $(P=0.36)$ but appeared to have a similar association with WURS scores. To further assess the dependence of the findings on the use of psychotropic medications, we constructed a dichotomous variable based on the use of antidepressants, antipsychotics, mood stabilizers, or stimulants and entered it as a covariate in the model. The results remained unchanged from that described above.

\section{Discussion}

This is the first study to estimate the prevalence of presumptive-ADHD in the geriatric population in the United States. The present results demonstrate that the frequency of ADHD tendency as assessed by the WURS is relatively stable across generations, even in the geriatric population. The prevalence of ADHD in children and adolescents is currently estimated at 3-9.5\% $[1,3,5,9]$, while previous studies have demonstrated a potentially lower prevalence in middle-aged adults at $1-6 \%[22,23,27]$. Our estimate of $4.4 \%$ lies well within the span of these ranges. The popular belief that ADHD is increasing could be due to a surveillance bias or to the increased development and utilization of screening tests such as the WURS in younger populations [8].

We did not find evidence of an increased frequency of WURS-positive, presumptive-ADHD in cognitively impaired individuals compared to cognitively normal controls, despite previous reports of an association with the diagnoses of MCI and early Alzheimer's disease in Down syndrome patients [28]. It is possible that this exploratory analysis failed to find a difference due to the low sample size in the cognitively impaired subjects studied. Further analysis of association of amnestic and nonamnestic presentations and WURS-positive ADHD symptoms are again precluded by the low number of subjects with WURS-positive ADHD in the sample, but should prove fertile ground for future research 
TABLE 3: Mean neuropsychological test scores $( \pm$ SEM $)$ in cognitively intact subjects only, adjusted for age, sex, and education in presumptive positive and negative childhood ADHD subjects as determined by Wender-Utah Rating Scale score.

\begin{tabular}{lccc}
\hline Neuropsychological test scores & ADHD positive $(n=10)$ & ADHD negative $(n=233)$ & $P$ value \\
\hline Folstein MMSE & $29.3 \pm 0.3$ & $29.2 \pm 0.1$ & 0.64 \\
Animal Fluency & $18.2 \pm 1.6$ & $20.9 \pm 0.4$ & $35.4 \pm 0.8$ \\
Trailmaking test A & $34.3 \pm 3.4$ & $80.5 \pm 1.9$ & 0.74 \\
Trailmaking test B & $82.8 \pm 8.5$ & $46.8 \pm 0.7$ & 0.79 \\
WAIS-R Digit-Symbol Substitution & $45.1 \pm 3.0$ & $14.0 \pm 0.3$ & 0.58 \\
WMS Logical Memory Delayed & $13.8 \pm 1.2$ & $9.6 \pm 0.1$ & 0.90 \\
WAIS-R Digit Span Forward & $8.5 \pm 0.6$ & $7.5 \pm 0.1$ & 0.07 \\
WAIS-R Digit Span Backward & $7.2 \pm 0.6$ & 0.61 \\
\hline
\end{tabular}

Abbreviations: MMSE: Mini-Mental State Examination; CDR: Clinical Dementia Rating scale; WAIS-R: Wechsler Adult Intelligence Scale-Revised; WMS: Wechsler Memory Scale.

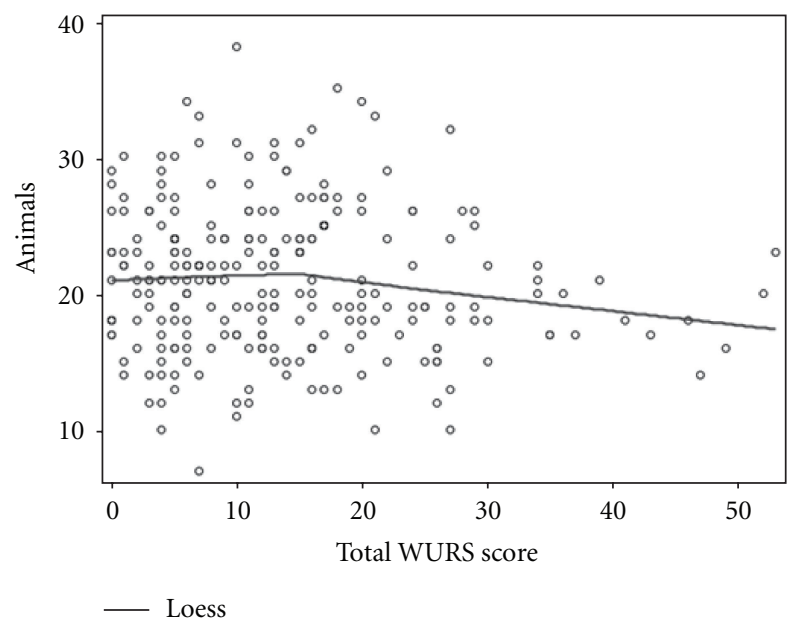

(a)

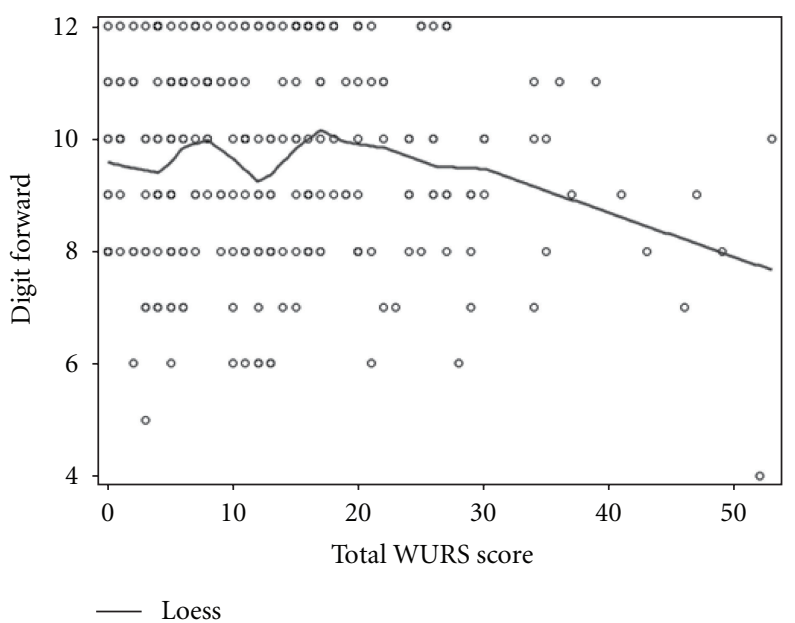

(b)

FIGURE 1: Scatterplots of $(\mathrm{a})$ animal naming total words $(P=0.030)$ and $(\mathrm{b})$ WAIS digit span total length $(P=0.037)$ versus WURS scores with LOESS fit using WURS scores squared as a quadratic term after adjustment for age and education. Data presented is for cognitively normal subjects only.

endeavors. One recent study from Argentina examined the Spanish WURS in normal elderly, AD, and dementia with Lewy bodies (DLB), demonstrating an association of higher WURS scores with DLB, but not AD [37]. A relatively high frequency of WURS-positive normal controls (15.1\%) calls this data into question and remains unexplained by the authors. Another study found increased tendencies for ADHD behaviors in adults with Down syndrome presenting with mild cognitive impairment or early Alzheimer's disease [28]. This study however did not include analysis of sporadic $\mathrm{AD}$ or the genetically normal geriatric population that is the focus of the present study. Definitive evidence of an association between childhood ADHD and the late life degenerative dementia is clearly lacking. An important caveat is that the lack of an association in the present study is based on purely cross sectional prevalence data, and so it is still possible that longitudinal analysis over time will show associations with the development of incident cognitive impairment. Such analyses from this and other cohorts will be important contributions to the literature in the future.
While the present data failed to demonstrate an association between WURS-positive, presumptive ADHD, and clinical diagnoses of MCI or dementia, they do demonstrate significant associations between tasks requiring sustained attention (Category Fluency and WAIS digit span tests; while category fluency is primarily a test of language fluency, normative performance is dependent on sustained attention to the task in addition to language function) and WURS scores in the normal elderly subjects studied. These results suggest that ADHD tendencies may represent lifelong cognitive profiles rather than transient early childhood and adolescent cognitive phenotypes. This interpretation is consistent with other studies that have demonstrated the persistence of ADHD tendencies into adulthood for many [22, 24, 25, 27]. It is also plausible that these findings represent a confound of recall bias where symptoms attributed to childhood performance are actually more representative of temporally proximate cognitive weaknesses. It should also be noted that only a subset of cognitive test scores were related to WURS scores, suggesting that cognitive deficits related to ADHD 
tendencies in the elderly may be restricted to specific cognitive domains or cognitive test paradigms and do not represent evidence for global cognitive decline necessary for a diagnosis of dementia using existing criteria.

The weaknesses of the present study include the effects of recall bias (potentially augmented by memory decline in the cognitively impaired subjects). Despite an awareness that amnestic symptoms are largely confined to short-term memory mechanisms in MCI and early-stage dementia (with relative sparing of long-term memories from childhood and early adult life) [38], it is quite possible that the reduced frequency of WURS-positive, presumptive-ADHD subjects in the impaired categories reflects early involvement of longterm in addition to short-term memory retrieval processes. The present study sought to overcome this confound by sampling both subject and informant data on all cognitively impaired subjects. Subject versus informant reports were significantly correlated, albeit with a moderate coefficient value. This was not unexpected in the 57 informants who responded who were mostly adult children and spouses of the participants and so did not actually know the subject personally during the early childhood and adolescent years. Thus, their responses on the WURS may well have been biased by the subjects' cognitive characteristics during early or middle-aged years or through second-hand information from the subjects themselves in years past. Despite these caveats, the finding of a positive association between subject and informant reports lends credence to the present use of subject reports on the WURS scores used as the basis for this analysis.

Weaknesses of the present study also include a potential for limited generalizability of the results. The UK ADC longitudinal cohort is largely Caucasian, highly educated and excludes participants with history of substance abuse and major psychiatric disorders (all factors that may lead to an underrepresentation of ADHD in the sample studied). A selection bias that may have underrepresented ADHD subjects cannot be excluded. Additional studies of ADHD tendencies using subjects with lower education levels and from other racial and ethnic backgrounds are needed to further validate and support any contention of generalizability in the present results. The lack of validation of the WURS with formal DSM-IV diagnoses of ADHD represents another weakness of the present study. Several previous studies have suggested high diagnostic accuracy (82-99\%), sensitivity (85-91\%), and specificity (76-91\%) of the WURS for the diagnosis of ADHD (confirmed by DSM-IV and or ICD-9 criteria) [33, 39-41]; however, other studies have suggested poor diagnostic accuracy in control subjects seeking evaluation for ADHD (57.5\% of the non-ADHD cases studied) and in control subjects with psychiatric diagnoses of depression $(66-81 \%)$ or bipolar disorder $(64 \%)$ [39, 41, 42]. These data suggest that the validity of the WURS is dependent on not only the presence of ADHD, but also other affective disorders. Exclusion criteria for the present group of subjects studied include the presence of any DSM-IV psychiatric diagnosis other than dementia, minimizing this confound, however, it is still possible that the frequency of WURSpositive, presumptive ADHD in this sample may have been inflated by the development of subclinical or undetected affective disorders in our population. Further studies in the geriatric population, with and without coexistent cognitive decline, will need to be validated against accepted DSM and ICD criteria.

The strengths of this study include the use of a clinically well-characterized, longitudinally followed cohort with extensive neuropsychological, neurological, and medical information readily available for analysis. All subjects in this cohort have also agreed to undergo autopsy and brain donation at death providing an additional opportunity in the future to study the potential pathological substrate(s) of ADHD tendencies. An additional strength of this study lies in its use of the previously validated WURS. Despite our use of a group of subjects older by several decades than those that served to previously validate the WURS, we did not encounter any difficulties when testing our cohort.

In summary, the present data represents a significant contribution to the field of aging research, representing the first such study of ADHD tendencies in the geriatric population and the association of childhood ADHD tendencies with late-life neurodegenerative cognitive impairment in the United States. These data suggest that (1) the frequency of WURS-positive, presumptive-ADHD appears stable across generations, (2) presumptive-ADHD is not associated with the diagnoses of MCI or dementia, and (3) the finding of deficits in attentional processing in presumptive-ADHD subjects in their geriatric years suggests that such traits are stable throughout life and need to be considered when interpreting cognitive test profiles in the elderly. Further studies in larger, more diverse populations are clearly needed to refine our understanding of the associations of ADHD with $\mathrm{MCI}$ or dementia diagnoses and the possible role childhood ADHD may play in the development of late-life cognitive decline.

\section{Conflict of Interests}

The authors report no conflict of interests.

\section{Acknowledgments}

This study was supported by funding from the NIH/NIA 1 P30 AG028383, 2R01AG019241, LRP 1 L30 AG032934, and the Sanders-Brown Foundation. The authors thank the many research participants who took the time to complete the study questionnaire and help move their understanding of aging and cognitive decline forward and Ms. Paula Thomason for editorial advice and formatting of this paper. The authors have nothing to disclose.

\section{References}

[1] W. J. Barbaresi, S. K. Katusic, R. C. Colligan et al., "How common is attention-deficit/hyperactivity disorder? Incidence in a population-based birth cohort in Rochester, Minn," Archives of Pediatrics and Adolescent Medicine, vol. 156, no. 3, pp. 217-224, 2002. 
[2] J. Biederman, "Attention-deficit/hyperactivity disorder: a selective overview," Biological Psychiatry, vol. 57, no. 11, pp. 1215-1220, 2005.

[3] E. J. Costello, S. Mustillo, A. Erkanli, G. Keeler, and A. Angold, "Prevalence and development of psychiatric disorders in childhood and adolescence," Archives of General Psychiatry, vol. 60, no. 8, pp. 837-844, 2003.

[4] P. Curatolo, C. Paloscia, E. D'Agati, R. Moavero, and A. Pasini, "The neurobiology of attention deficit/hyperactivity disorder," European Journal of Paediatric Neurology, vol. 13, no. 4, pp. 299-304, 2009.

[5] C. J. Kratochvil, B. S. Vaughan, A. Barker, L. Corr, A. Wheeler, and V. Madaan, "Review of pediatric attention deficit/hyperactivity disorder for the general psychiatrist," Psychiatric Clinics of North America, vol. 32, no. 1, pp. 39-56, 2009.

[6] E. Mick, S. V. Faraone, and J. Biederman, "Age-dependent expression of attention-deficit/hyperactivity disorder symptoms," Psychiatric Clinics of North America, vol. 27, no. 2, pp. 215-224, 2004.

[7] American Psychiatric Association, Diagnostic and Statistical Manual of Mental Disorders, American Psychiatric Association, Washington, DC, USA, 1994.

[8] I. Bitter, V. Simon, S. Bálint, A. Mészáros, and P. Czobor, "How do different diagnostic criteria, age and gender affect the prevalence of attention deficit hyperactivity disorder in adults? An epidemiological study in a Hungarian community sample," European Archives of Psychiatry and Clinical Neuroscience, vol. 260, no. 4, pp. 287-296, 2010.

[9] "Increasing prevalence of parent-reported attention-deficit/ hyperactivity disorder among children-United States, 2003 and 2007," Morbidity and Mortality Weekly Report (MMWR), vol. 59, pp. 1439-1443, 2010.

[10] D. A. Christakis, B. E. Ebel, F. P. Rivara, and F. J. Zimmerman, "Television, video, and computer game usage in children under 11 years of age," Journal of Pediatrics, vol. 145, no. 5, pp. 652-656, 2004.

[11] D. A. Christakis, F. J. Zimmerman, D. L. DiGiuseppe, and C. A. McCarty, "Early television exposure and subsequent attentional problems in children," Pediatrics, vol. 113, no. 4, pp. 708-713, 2004.

[12] H. C. Steinhausen, "The heterogeneity of causes and courses of attention-deficit/hyperactivity disorder," Acta Psychiatrica Scandinavica, vol. 120, no. 5, pp. 392-399, 2009.

[13] R. Taurines, J. Schmitt, T. Renner, A. C. Conner, A. Warnke, and M. Romanos, "Developmental comorbidity in attentiondeficit/hyperactivity disorder," ADHD Attention Deficit and Hyperactivity Disorders, vol. 2, no. 4, pp. 267-289, 2010.

[14] T. E. Wilens and T. J. Spencer, "Understanding attentiondeficit/hyperactivity disorder from childhood to adulthood," Postgraduate Medicine, vol. 122, no. 5, pp. 97-109, 2010.

[15] E. G. Willcutt, B. F. Pennington, L. Duncan et al., "Understanding the complex etiologies of developmental disorders: behavioral and molecular genetic approaches," Journal of Developmental and Behavioral Pediatrics, vol. 31, no. 7, pp. 533-544, 2010.

[16] A. Lo-Castro, E. D'Agati, and P. Curatolo, "ADHD and genetic syndromes," Brain and Development, vol. 33, no. 6, pp. 456461, 2010.

[17] S. V. Faraone and E. Mick, "Molecular genetics of attention deficit hyperactivity disorder," Psychiatric Clinics of North America, vol. 33, no. 1, pp. 159-180, 2010.

[18] T. Banaschewski, K. Becker, S. Scherag, B. Franke, and D. Coghill, "Molecular genetics of attention-deficit/hyperactivity disorder: an overview," European Child and Adolescent Psychiatry, vol. 19, no. 3, pp. 237-257, 2010.

[19] S. Bálint, P. Czobor, S. Komlósi, Á. Mészáros, V. Simon, and I. Bitter, "Attention deficit hyperactivity disorder (ADHD): gender- and age-related differences in neurocognition," Psychological Medicine, vol. 39, no. 8, pp. 1337-1345, 2009.

[20] J. Biederman, "Impact of comorbidity in adults with attention-deficit/hyperactivity disorder," Journal of Clinical Psychiatry, vol. 65, no. 3, supplement, pp. 3-7, 2004.

[21] S. V. Faraone, T. J. Spencer, B. Montano, and J. Biederman, "Attention-deficit/hyperactivity disorder in adults: a survey of current practice in psychiatry and primary care," Archives of Internal Medicine, vol. 164, no. 11, pp. 1221-1226, 2004.

[22] J. Fayyad, R. De Graaf, R. Kessler et al., "Cross-national prevalence and correlates of adult attention-deficit hyperactivity disorder," British Journal of Psychiatry, vol. 190, pp. 402-409, 2007.

[23] R. C. Kessler, L. Adler, M. Ames et al., "The prevalence and effects of adult attention deficit/hyperactivity disorder on work performance in a nationally representative sample of workers," Journal of Occupational and Environmental Medicine, vol. 47, no. 6, pp. 565-572, 2005.

[24] R. C. Kessler, L. A. Adler, R. Barkley et al., "Patterns and predictors of attention-deficit/hyperactivity disorder persistence into adulthood: results from the national comorbidity survey replication," Biological Psychiatry, vol. 57, no. 11, pp. 14421451, 2005.

[25] S. Mannuzza, R. G. Klein, A. Bessler, P. Malloy, and M. Lapadula, "Adult psychiatric status of hyperactive boys grown up," American Journal of Psychiatry, vol. 155, no. 4, pp. 493498, 1998.

[26] V. Simon, P. Czobor, S. Balint, A. Meszaros, and I. Bitter, "Prevalence and correlates of adult attention-deficit hyperactivity disorder: meta-analysis," British Journal of Psychiatry, vol. 194, no. 3, pp. 204-211, 2009.

[27] T. E. Wilens, S. V. Faraone, and J. Biederman, "Attentiondeficit/hyperactivity disorder in adults," Journal of the American Medical Association, vol. 292, no. 5, pp. 619-623, 2004.

[28] S. J. Krinsky-McHale, D. A. Devenny, P. Kittler, and W. Silverman, "Selective attention deficits associated with mild cognitive impairment and early stage Alzheimer's disease in adults with Down syndrome," American Journal on Mental Retardation, vol. 113, no. 5, pp. 369-419, 2008.

[29] A. M. Boonstra, J. Oosterlaan, J. A. Sergeant, and J. K. Buitelaar, "Executive functioning in adult ADHD: a metaanalytic review," Psychological Medicine, vol. 35, no. 8, pp. 1097-1108, 2005.

[30] D. W. Lovejoy, J. D. Ball, M. Keats et al., "Neuropsychological performance of adults with attention deficit hyperactivity disorder (ADHD): diagnostic classification estimates for measures of frontal lobe/executive functioning," Journal of the International Neuropsychological Society, vol. 5, no. 3, pp. 222233, 1999.

[31] L. J. Seidman, A. Doyle, R. Fried, E. Valera, K. Crum, and L. Matthews, "Neuropsychological function in adults with attention-deficit/hyperactivity disorder," Psychiatric Clinics of North America, vol. 27, no. 2, pp. 261-282, 2004.

[32] G. S. Smith, E. Kramer, Y. Ma et al., "The functional neuroanatomy of geriatric depression," International Journal of Geriatric Psychiatry, vol. 24, no. 8, pp. 798-808, 2009.

[33] M. F. Ward, P. H. Wender, and F. W. Reimherr, "The Wender Utah Rating Scale: an aid in the retrospective diagnosis of childhood attention deficit hyperactivity disorder," American Journal of Psychiatry, vol. 150, no. 6, pp. 885-890, 1993. 
[34] F. A. Schmitt, M. M. C. Wetherby, D. R. Wekstein, C. M. S. Dearth, and W. R. Markesbery, "Brain donation in normal aging: procedures, motivations, and donor characteristics from the Biologically Resilient Adults in Neurological Studies (BRAiNS) project," Gerontologist, vol. 41, no. 6, pp. 716-722, 2001.

[35] M. F. Folstein, S. E. Folstein, and P. R. McHugh, “'Mini mental state. A practical method for grading the cognitive state of patients for the clinician," Journal of Psychiatric Research, vol. 12, no. 3, pp. 189-198, 1975.

[36] J. C. Morris, “The Clinical Dementia Rating (CDR): current version and scoring rules," Neurology, vol. 43, no. 11, pp. 24122414, 1993.

[37] A. Golimstok, J. I. Rojas, M. Romano, M. C. Zurru, D. Doctorovich, and E. Cristiano, "Previous adult attentiondeficit and hyperactivity disorder symptoms and risk of dementia with Lewy bodies: a case-control study," European Journal of Neurology, vol. 18, no. 1, pp. 78-84, 2011.

[38] G. A. Jicha and S. A. Carr, "Conceptual evolution in Alzheimer's disease: implications for understanding the clinical phenotype of progressive neurodegenerative disease," Journal of Alzheimer's Disease, vol. 19, no. 1, pp. 253-272, 2010.

[39] B. Oncü, S. Olmez, and V. Sentürk, "Validity and reliability of the Turkish version of the Wender Utah Rating Scale for attention-deficit/hyperactivity disorder in adults," Türk Psikiyatri Dergisi, vol. 16, no. 4, pp. 252-259, 2005.

[40] P. Retz-Junginger, W. Retz, D. Blocher et al., "Reliability and validity of the German short version of the Wender-Utah Rating Scale for the retrospective assessment of attention deficit/hyperactivity disorder," Nervenarzt, vol. 74, no. 11, pp. 987-993, 2003.

[41] R. Rodriguez-Jimenez, G. Ponce, R. Monasor et al., "Validation in the adult Spanish population of the Wender Utah Rating Scale for the retrospective evaluation in adults of attention deficit/hyperactivity disorder in childhood," Revista de Neurología, vol. 33, pp. 138-144, 2001.

[42] B. S. McCann, L. Scheele, N. Ward, and P. Roy-Byrne, "Discriminant validity of the Wender Utah Rating Scale for attention-deficit/hyperactivity disorder in adults," Journal of Neuropsychiatry and Clinical Neurosciences, vol. 12, no. 2, pp. 240-245, 2000. 


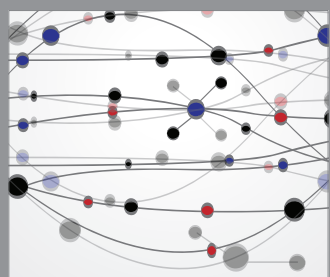

The Scientific World Journal
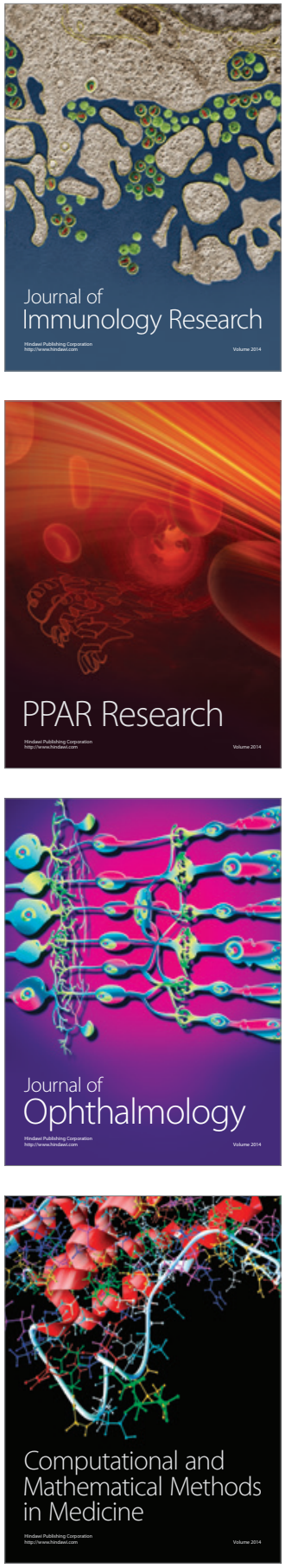

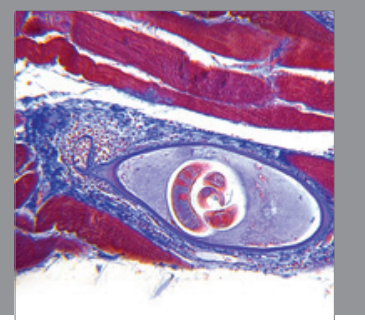

Gastroenterology

Research and Practice
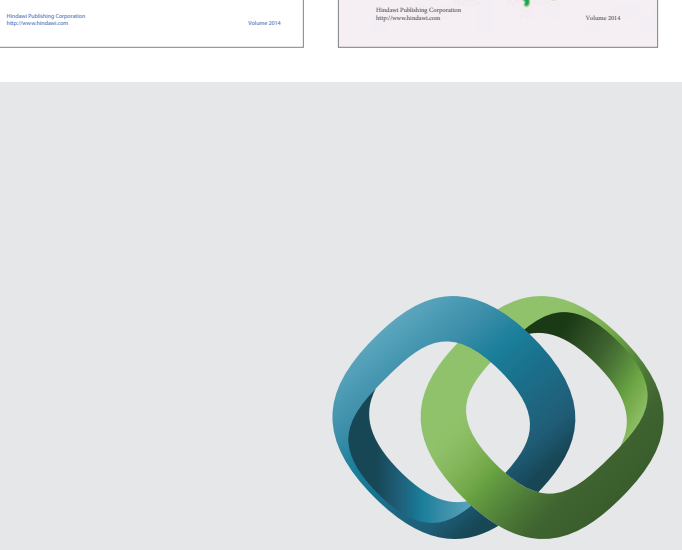

\section{Hindawi}

Submit your manuscripts at

http://www.hindawi.com
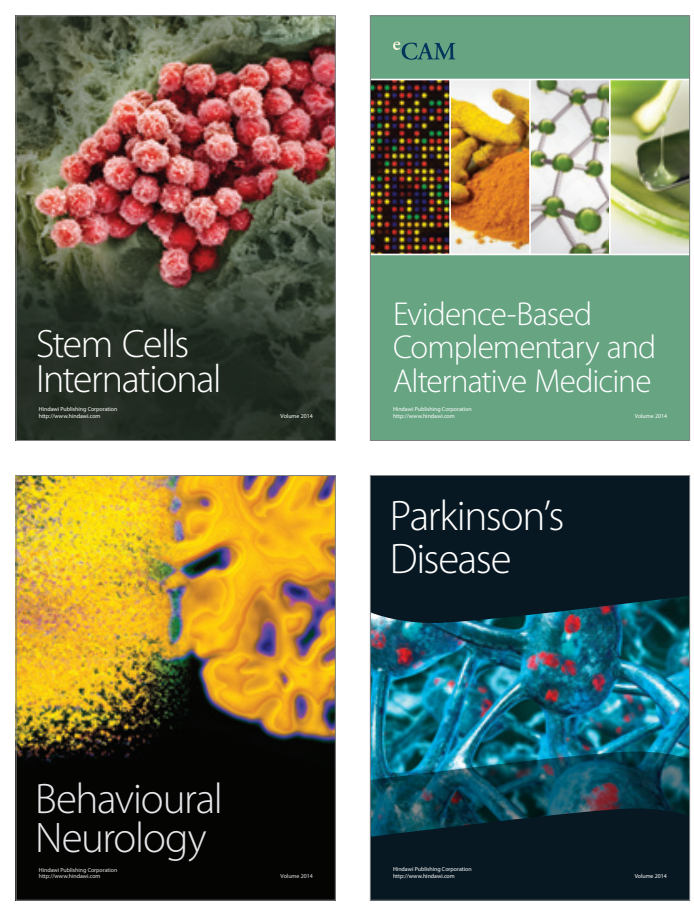

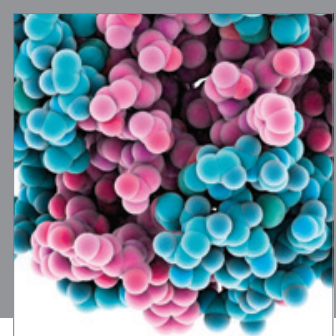

Journal of
Diabetes Research

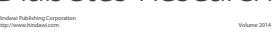

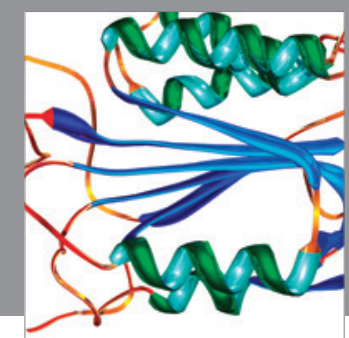

Disease Markers
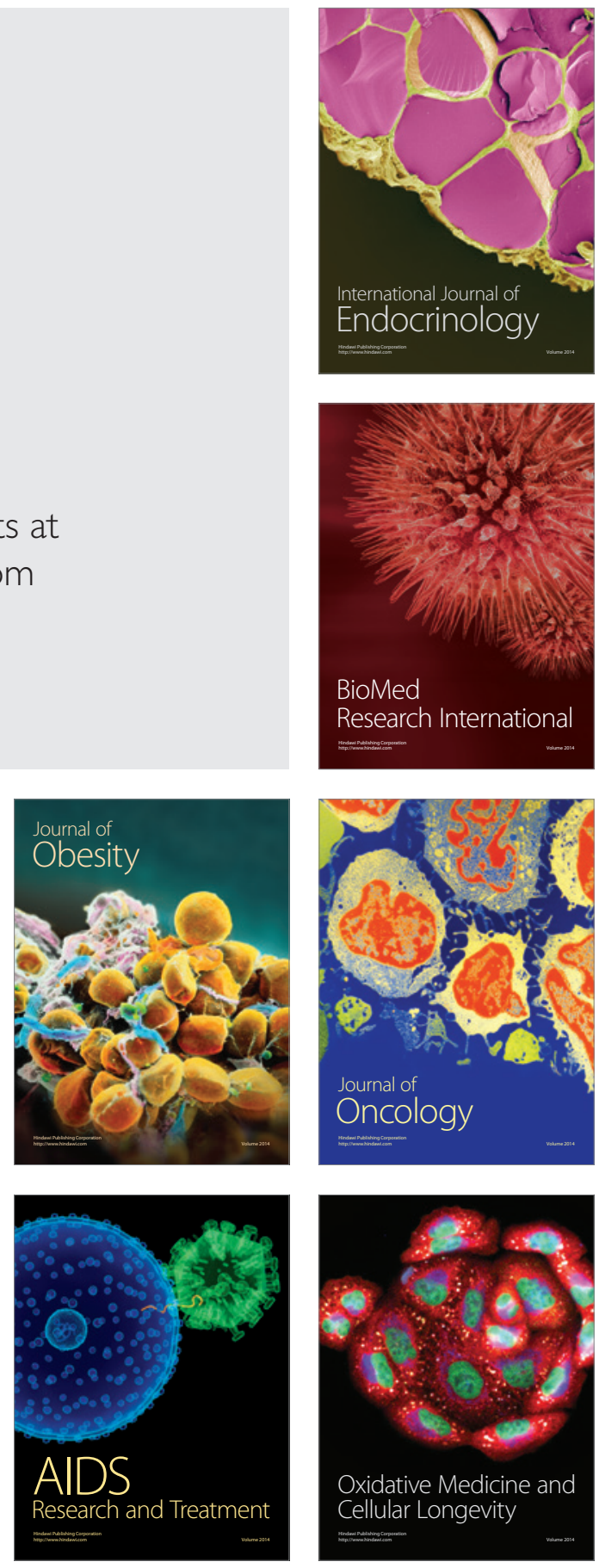